Pacific Journal of Mathematics

THE INVARIANCE PRINCIPLE FOR WAVE OPERATORS 


\section{THE INVARIANCE PRINCIPLE FOR WAVE OPERATORS}

\section{MANFRED WOLLENBERG}

The invariance principle for wave operators is proved. It is shown that the existence of wave operators $W_{ \pm}(B, A)$ does not imply the existence of $W_{ \pm}(g(B), g(A))$, in general.

1. Introduction. Let $A$ and $B$ be two selfadjoint operators on a separable Hilbert space $\mathscr{H}$ and let $P_{A}$ and $P_{B}$ be the orthogonal projections on the spaces of absolute continuity for $A$ and $B$, respectively. The wave operators $W_{ \pm}(B, A)$ are defined by the strong limits

$$
W_{ \pm}(B, A) \equiv s-\lim _{t \rightarrow \pm \infty} e^{i t B} e^{-i t A} P_{A}
$$

when they exist (cf. [2, Chapter X]). The invariance principle of M. S. Birman and T. Kato says: If the wave operators $W_{ \pm}(B, A)$ and $W_{ \pm}(g(B), g(A))$ exist and $g(\lambda)$ is real-valued and piecewise monotone increasing, with a certain mild smoothness, then

$$
W_{ \pm}(g(B), g(A))=W_{ \pm}(B, A) .
$$

As stated by T. Kato and S. T. Kuroda in [3]: "It would be nice if the existence of $W_{ \pm}(B, A)$ implied the existence of $W_{ \pm}(g(B)$, $g(A))$ and the invariance principle.

However, this has not been shown in general".

For example, the existence of $W_{ \pm}(g(B), g(A))$ and the invariance principle have been proved under the condition that $B-A$ or $(B-$ $\xi)^{-1}-(A-\xi)^{-1}(\xi$ a nonreal number) is a trace-class operator (see for instance [2, Chapter X]).

The aim of this paper is

1. the proof of the invariance principle for wave operators,

2. the proof, that the existence of $W_{ \pm}(B, A)$ does not imply the existence of $W_{ \pm}(g(B), g(A))$, in general.

In the present work we restrict our considerations to real-valued functions $g(\lambda)$ on $(-\infty, \infty)$ with the following properties (cf. [2, p. 543]): The whole interval $(-\infty, \infty)$ can be divided into a countable number of subintervals $\Delta_{n}$ with lengths $l_{n}$ in such a way that min $l_{n}>0$ and in each open subinterval $g(\lambda)$ is differentiable with $g^{\prime}(\lambda)$ 
continuous, locally of bounded variation, and positive. A function with these properties is called an allowable function. Furthermore, we shall consider only the wave operators $W_{+}$because the theorems and proofs for the wave operators $W_{\text {- }}$ are entirely similar. In $\S 2$ we prove

THEOREM 1 (invariance principle). Let $A$ and $B$ be two selfadjoint operators on a separable Hilbert space $\mathscr{H}$ and let $g(\lambda)$ be an allowable function. If $W_{+}(B, A)$ and $W_{+}(g(B), g(A))$ exist and if $W_{+}(B, A)$ is complete, then $W_{+}(g(B), g(A))=W_{+}(B, A)$.

From Theorem 1 we also see that the existence of $W_{+}(B, A)$, $W_{+}(g(B), g(A))$ and the completeness of $W_{+}(B, A)$ imply the completeness of $W_{+}((g, B), g(A))$. The next two theorems concern the existence of the wave operator $W_{+}(g(B), g(A))$. They will be proved in $\S \S 2$ and 3 , respectively.

THEOREM 2. Let $A$ and $B$ be two selfadjoint operators on $a$ separable Hilbert space $\mathscr{H}$ with the absolutely continous spectrum $\Lambda$ and let $g(\lambda)$ be an allowable function. If the wave operator $W_{+}(B, A)$ exists, is complete and if $g(\lambda)$ is piecewise linear on $\Lambda$, then $W_{+}(g(B), g(A))$ exists.

THEOREM 3. Let $A$ be a selfadjoint operator on a separable Hilbert space $\mathscr{H}$ with the absolutely continuous spectrum $\Lambda \neq 0$. Let $g(\lambda)$ be an allowable function for which a finite interval $\Delta \subset(-\infty$, $\infty$ ) with $|\Delta \cap \Lambda| \neq 0$ (Lebesgue measure) exists such that on $\Delta g^{\prime}(\lambda)$ exists and is a continuous strictly monotone function. Then there $i$ is a selfadjoint operator $B$ such that $W_{+}(B, A)$ exists, is complete, however, $W_{+}(g(B), g(A))$ does not exist.

It is easily seen, for instance, that all allowable functions $g(\lambda)$ which are piecewise twice continuously differentiable satisfy the assumptions either of Theorem 2 or of Theorem 3 for fixed $\Lambda$.

For the proofs of the theorems we use the following result of H. Baumgärtel [1, Theorem 3]:

(NS) Let $W$ be a partial isometry with

$$
W^{*} W=P_{A}, W W^{*}=P_{B}, W A P_{A} W^{*} \supseteqq B P_{B} .
$$

Then $W_{+}(B, A)$ exists and $W=W_{+}(B, A)$ if and only if

$$
\begin{gathered}
W=P_{A}+C, \\
s-\lim _{t \rightarrow \infty} e^{i t A} C e^{-i t A} P_{A}=0 .
\end{gathered}
$$


By Theorem 1 we obtain from (NS) that the existence and completeness of $W_{+}(B, A)$ imply the existence of $W_{+}(g(B), g(A))$ if and only if for the operator $C$ defined by (1.3) the strong limit

$$
s-\lim _{t \rightarrow \infty} e^{i t g(A)} C e^{-i t g(A)} P_{A}=0
$$

exists. Here it was used that $P_{g(A)}=P_{A}$ for allowable functions $g(\lambda)$ (see $\S 2$ ).

Hence we know that the proof of Theorem 3 leads to the construction of an operator $C$ for which $s-\lim _{t \rightarrow \infty} e^{i t A} C e^{-i t A} P_{A}=0$ and $s-\lim _{t \rightarrow \infty} e^{i t g(A)} C e^{-i t g(A)} P_{A}$ does not exist for the function $g(\lambda)$ defined by Theorem 3. To prove Theorem 2 we shall show that the equation

$$
s-\lim _{t \rightarrow \infty} e^{i t A} C e^{-i t A} P_{A}=0
$$

implies $s-\lim _{t \rightarrow \infty} e^{i t g(A)} C e^{-i t g(A)} P_{A}=0$ for piecewise linear functions $g(\lambda)$. The invariance principle will be proved by means of

LEMMA 1. Let $T$ be a nonnegative bounded selfadjoint operator and $g(\lambda)$ an allowable function. If the strong limits

$$
s-\lim _{t \rightarrow \infty} e^{i t A} T e^{-i t A} P_{A}=0, s-\lim _{t \rightarrow \infty} e^{i t g(A)} T e^{-i t g(A)} P_{A}
$$

exist, then they are equal.

In $\S 5$ we prove Lemma 1 and formulate and prove two other lemmas which concern the behavior of the function $e^{-i t g(\lambda)}$ for large $t$.

2. Proof of Theorem 1. First we introduce several notations and simple relations which are needed for the proof. As in $\S 1$ let $H$ be a selfadjoint operator on a separable Hilbert space $\mathscr{H}$ and $P_{H}$ be the orthogonal projection on the space of absolute continuity. We note that for every allowable function $g(\lambda)$

$$
P_{g(H)}=P_{H} \text {. }
$$

(2.1) has been proved in [2, p. 545] for a class of functions slightly more restrictive than the allowable functions. The proof can easily be generalized for all allowable functions. Furthermore, we introduce the notations $\{H\}^{\prime}$ for the commutant of $H$ and

$$
V_{H}^{+}(X) \equiv s-\lim _{t \rightarrow \infty} e^{i t H} X e^{-i t H} P_{H},
$$

whenever for the bounded operator $X$ the strong limit exists. If $V_{H}^{+}(X)$ for the bounded operator $X$ exists, then we have the unambiguous decomposition (cf. [1]) 


$$
X=X_{1}+X_{2}
$$

where

$$
\begin{gathered}
X_{1}=P_{H} X_{1}=X_{1} P_{H} \in\{H\}^{\prime}, \\
V_{H}^{+}\left(X_{2}\right)=0 .
\end{gathered}
$$

For continuous functions $f(\lambda)$ and a selfadjoint operator $X$ one easily verifies that

$$
f\left(V_{H}^{+}(X)\right)=V_{H}^{+}(f(X)) .
$$

Now we prove Theorem 1 . By (NS), we find that $W_{+}(B, A)=$ $P_{A}+C$ with $V_{A}^{+}(C)=0$. Further we also have

$$
V_{A}^{+}\left(C^{*}\right)=0,
$$

since

$$
\begin{aligned}
V_{A}^{+}\left(C^{*}\right) & =V_{A}^{+}\left(C^{*}+P_{A}\right)-P_{A}=V_{A}^{+}\left(W_{+}^{*}(B, A)\right)-P_{A} \\
& =s-\lim _{t \rightarrow \infty} e^{i t A} W_{+}^{*}(B, A) e^{-i t A} P_{A}-P_{A} \\
& =s-\lim _{t \rightarrow \infty} W_{+}^{*}(B, A) e^{i t B} e^{-i t A} P_{A}-P_{A} \\
& =W_{+}^{*}(B, A) W_{+}(B, A)-P_{A}=0
\end{aligned}
$$

with the intertwining relation $W_{+}(B, A) e^{i t A}=e^{i t B} W_{+}(B, A)$. We define (2.8)

$$
W_{1} \equiv W_{+}^{*}(B, A) W_{+}(g(B), g(A)) \text {. }
$$

From this definition we obtain that $V_{g(A)}^{+}\left(W_{+}^{*}(B, A)\right)$ exists and

$$
V_{g(A)}^{+}\left(W_{+}^{*}(B, A)\right)=W_{1},
$$

since

$$
\begin{gathered}
V_{g(A)}^{+}\left(W_{+}^{*}(B, A)\right)=s-\lim _{t \rightarrow \infty} e^{i t g(A)} W_{+}^{*}(B, A) e^{-i t g(A)} P_{A} \\
=s-\lim _{t \rightarrow \infty} W_{+}^{*}(B, A) e^{i t g(B)} e^{-i t g(A)} P_{A}=W_{1} .
\end{gathered}
$$

By (2.3) and (2.5) then we have

$$
W_{+}^{*}(B, A)=W_{1}+C_{1}
$$

with

$$
\begin{gathered}
V_{g(A)}^{+}\left(C_{1}\right)=0 \\
W_{1}=P_{A} W_{1}=W_{1} P_{A} \in\{g(A)\}^{\prime} .
\end{gathered}
$$

From the Definition (2.8) and the completeness of $W_{+}(B, A)$ one 
easily verifies that $W_{1}$ is $a$ partially isometrie with

$$
W_{1}^{*} W_{1}=P_{A}, W_{1} W_{1}^{*}=P_{1} \leqq P_{A} \text {. }
$$

Further we have

$$
V_{g(A)}^{+}\left(C_{1}^{*} P_{1}\right)=0
$$

This follows from

$$
\begin{aligned}
& V_{g(A)}^{+}\left(C_{1}^{*} P_{1}\right)=0 \longleftrightarrow V_{g(A)}^{+}\left(W_{+}^{*}(B, A) C_{1}^{*} P_{1}\right) \\
& \quad=V_{g(A)}^{+}\left(W_{+}^{*}(B, A) C_{1}^{*} P_{1}+C_{1} W_{1}^{*}+P_{1}\right)-P_{1} \\
& \quad=V_{g(A)}^{+}\left(W_{+}^{*}(B, A)\left(C_{1}^{*}+W_{1}^{*}\right) P_{1}\right)-P_{1} \\
& \quad=V_{g(A)}^{+}\left(W_{+}^{*}(B, A) W_{+}(B, A) P_{1}\right)-P_{1}=0
\end{aligned}
$$

with $V_{g(A)}^{+}\left(C_{1} W_{1}^{*}+P_{1}\right)=P_{1}$ by $W_{1}^{*}, P_{1} \in\{g(A)\}^{\prime}$. Combining (2.10) with $W_{+}^{*}(B, A)=P_{A}+C^{*}$ and (2.13) we obtain

$$
\begin{aligned}
C P_{1} C^{*}= & \left(W_{1}+C_{1}-P_{A}\right)^{*} P_{1}\left(W_{1}+C_{1}-P_{A}\right) \\
= & \left(W_{1}-P_{1}\right)^{*}\left(W_{1}-P_{1}\right)+\left(W_{1}-P_{1}\right)^{*} C_{1}+C_{1}^{*} P_{1}\left(W_{1}-P_{1}\right) \\
& +C_{1}^{*} P_{1} C_{1}=\left(W_{1}-P_{1}\right)^{*}\left(W_{1}-P_{1}\right)+C_{2} .
\end{aligned}
$$

By (2.11), (2.14) and $\left(W_{1}-P_{1}\right),\left(W_{1}-P_{1}\right)^{*} \in\{g(A)\}^{\prime}$ we have $V_{g(A)}^{+}\left(C_{2}\right)=$ 0 and therefore,

$$
V_{g(A)}^{+}\left(C P_{1} C^{*}\right)=\left(W_{1}-P_{1}\right)^{*}\left(W_{1}-P_{1}\right) .
$$

Furthermore, it follows from (2.7) that

$$
V_{A}^{+}\left(C P_{1} C^{*}\right)=0 \text {. }
$$

The operator $C P_{1} C^{*}$ satisfies the assumptions of Lemma 1 . Hence, we have $\left(W_{1}-P_{1}\right) *\left(W_{1}-P_{1}\right)=0$ and also $\left(W_{1}-P_{1}\right)=0$. With (2.13) and (2.8), we finally obtain $W_{1}=P_{A}$ and

$$
W_{+}(g(B), g(A))=W_{+}(B, A) \text {. }
$$

3. Proof of Theorem 2. We shall use the same notations as in §2. By Theorem 1 and (NS) it is necessary and sufficient for the existence of $W_{+}(g(B), g(A))$ that

$$
V_{g(A)}^{+}(C)=0 .
$$

Let $\varphi \in P_{A} \mathscr{H}, \varepsilon>0$ and $P_{A}(\Delta)$ be the spectral measure of $A$. Then there is a finite interval $\Delta^{\prime}$ such that

$$
\left\|\varphi-P_{\Delta}\left(\Delta^{\prime}\right) \varphi\right\| \leqq \frac{\varepsilon}{3\|C\|} .
$$


From the definition of the functions $g(\lambda)$ in Theorem 2 we see that there exists a finite number of disjoint intervals $\Delta_{n} \subset \Delta^{\prime}(n=1,2, \cdots$, $N)$ such that

$$
\begin{gathered}
g(A) P_{A} P_{A}\left(\Delta_{n}\right)=q_{n} A P_{A} P_{A}\left(\Delta_{n}\right), \\
\left\|P_{A}\left(\Delta^{\prime}\right) \varphi-\sum_{n=1}^{N} P_{A}\left(\Delta_{n}\right) \varphi\right\| \leqq \frac{\varepsilon}{3\|C\|}
\end{gathered}
$$

with $0<q_{n}<\infty$. By (3.2), (3.3), and (3.4) we find

$$
\left\|C e^{-i t g(A)} P_{A} \varphi\right\| \leqq \frac{2}{3} \varepsilon+\sum_{n=1}^{N}\left\|C e^{-i t q_{n} A} P_{A}\left(\Delta_{n}\right) \varphi\right\| .
$$

$C$ satisfies the relation (see (NS))

$$
\left\|C e^{-i t a} P_{A} \psi\right\| \longrightarrow 0 \text { as } t \longrightarrow \infty
$$

for every $\psi \in \mathscr{H}$. Hence, for the functions $\varphi_{n}=P_{A}\left(\Delta_{n}\right) \varphi$ there are numbers $T_{n}$ such that

$$
\left\|C e^{-i t q_{n}{ }^{A}} \Phi_{n}\right\| \leqq \frac{\varepsilon}{3 N} \quad \text { for all } t>T_{n} .
$$

By (3.5), (3.9) we obtain

$$
\left\|C e^{-i t g(A)} P_{A} \varphi\right\| \leqq \varepsilon \text { for } t>T=\max _{n} T_{n}
$$

and (3.1) is proved.

4. Proof of Theorem 3. For simplicity we shall assume that $\Delta \subset \Lambda$ and $\Delta=[0,2 \pi]$. Let $u \in P_{A}(\Delta) P_{A} \mathscr{H}$ with $P_{A}\left(\Delta^{\prime}\right) u \neq 0$ for all $\Delta^{\prime} \subset \Delta,\left|\Delta^{\prime}\right| \neq 0$. A restricted on the subspace $\mathscr{H}_{1}=\overline{\operatorname{sp}}\left\{P_{A}\left(\Delta^{\prime}\right) u, \Delta^{\prime} \subset \Delta\right.$ Borel set\} is an operator with simple absolutely continuous spectrum $\Delta$. Hence we may identify $\mathscr{H}_{1}$ with $\mathscr{L}^{2}(\Delta)$ and $a$ restricted on $\mathscr{H}_{1}$ with the multiplication operator by $\lambda$ on $\mathscr{L}^{2}(\Delta)$ denoted by $H$.

Therefore it follows that for the proof of Theorem 3 it is sufficient to show that for $H$ such an operator $B$ defined by Theorem 3 exists. At first we construct a projector $P$ such that $V_{H}^{+}(P)=0$ and $V_{g(H)}^{+}(P)$ do not exist. We consider the function

$$
\frac{1}{\sqrt{2 \pi}} e^{-i t g(\lambda)}=\sum_{m} \psi_{t}^{m} \frac{1}{\sqrt{2 \pi}} e^{-i \lambda m} \in \mathscr{L}^{2}(\Delta) .
$$

By Lemma 2 for $\varepsilon=1 / 2$ we can find sequences of natural numbers $S_{n}, N_{n}$ with $S_{n}, N_{n} \rightarrow \infty$ as $n \rightarrow \infty$ such that

$$
\sum_{m=N_{n}}^{N_{n+1}-1}\left|\psi_{s_{n}}^{m}\right|^{2}=a_{n} \geqq 1-\varepsilon=\frac{1}{2} \text {. }
$$


Now we define $P$ by

$$
\begin{gathered}
P=\sum_{n=1}^{\infty} f_{n}\left(\cdot, f_{n}\right), \\
f_{n}(\lambda)=\sum_{m=N_{n}}^{N_{n+1}-1} \frac{1}{\sqrt{a_{n}}} \psi_{S_{n}}^{m} \frac{1}{\sqrt{2 \pi}} e^{-i \lambda m} .
\end{gathered}
$$

Next we prove $V_{H}^{+}(P)=0$, i.e.,

$$
\lim _{t \rightarrow \infty}\left\|P e^{-i t H} \psi\right\|=0 \text { for every } \psi \in \mathscr{L}^{2}(\Delta)
$$

As is easily shown, for the proof of (4.4) it is sufficient to consider the sequence $\left\|P e^{-i n H} \psi_{0}\right\|$ with $n \rightarrow \infty$ ( $n a$ natural number) and $\psi_{0}=$ $1 / \sqrt{2 \pi}$.

We have

$$
\begin{aligned}
\left\|P e^{-i p H} \psi_{0}\right\|^{2} & =\sum_{n=1}^{\infty}\left|\left(f_{n}, e^{-i p H} \psi_{0}\right)\right|^{2} \\
& =\sum_{n=1}^{\infty}\left|\frac{1}{\sqrt{2 \pi}} \int_{0}^{2 \pi} f_{n}(\lambda) e^{i p \lambda} d \lambda\right|^{2} \\
& =\sum_{n=1}^{\infty}\left|\sum_{m=N_{n}}^{N_{n+1}-1} \psi_{S_{n}}^{m} \frac{1}{\sqrt{a_{n}}} \delta_{m ; p}\right|^{2} \\
& =\frac{\Lambda}{a_{x(p)}}\left|\psi_{S x(p)}^{p}\right|^{2},
\end{aligned}
$$

where $x(p)=r$ if $p \in\left(N_{r}, N_{r}+1, \cdots, N_{r+1}-1\right)$.

It is clear that $x(p) \rightarrow \infty$ as $p \rightarrow \infty$. Since $g(\lambda)$ satisfies the assumptions of Lemma 3 we find $\left|\psi_{s x(p)}^{p}\right|^{2} \rightarrow 0$ as $p \rightarrow \infty$ and also $\left\|P e^{-i p H} \psi_{0}\right\|^{2} \rightarrow 0$ as $p \rightarrow \infty$. This proves (4.4). To prove that $V_{o(H)}^{+}(P)$ does not exist by Lemma 1 it is sufficient to show that there are a $\psi \in \mathscr{L}^{2}(\Delta)$, a sequence of real numbers $t_{n} \rightarrow \infty$ as $n \rightarrow \infty$ and an $X>0$ such that

$$
\left\|P e^{-i t_{n} \cdot g(H)} \psi\right\|>X \text { for all } t_{n} .
$$

We set $\psi=\psi_{0}=1 / \sqrt{2 \pi}$ and $t_{n}=S_{n}$ (see (4.1)). Then by (4.1), (4.2)

$$
\begin{aligned}
& \left\|P e^{-i S_{n} \cdot g(H)} \psi_{0}\right\|^{2}=\sum_{q=1}^{\infty}\left|\left(f_{q}, e^{-i S_{n} \cdot g(H)} \psi_{0}\right)\right|^{2} \\
& \quad \geqq\left|\left(f_{n}, e^{-i S_{n} \cdot g(H)} \psi_{0}\right)\right|^{2}=\left|\frac{1}{\sqrt{a_{n}}} \sum_{m=N_{n}}^{N_{n+1}-1} \psi_{S_{n}}^{m} \frac{1}{\sqrt{2 \pi}} \int_{0}^{2 \pi} d \lambda e^{i S_{n} \cdot g(\lambda)} e^{-i m \lambda}\right|^{2} \\
& \quad=\left.\left|\frac{1}{\sqrt{a_{n}}} \sum_{m=N_{n}}^{N_{n+1}-1}\right| \psi_{S_{n}}^{m}\right|^{2}=a_{n} \geqq \frac{1}{2} \text { for all } S_{n} .
\end{aligned}
$$

This proves that $U_{g(H)}^{+}(P)$ does not exist.

Now we define by $U \equiv 1-2 P$ a unitary operator and we set 
$B=U H U$. From the definitions of $U, B$, and (NS) it immediately follows that $W_{+}(B, H)$ exists and $U=W_{+}(B, H)$. Since, however, $V_{g(H)}^{+}(-2 P)$ does not exist, it follows from Theorem 1 and (NS) that also $W_{+}(g(B), g(H))$ does not exist.

5. Proofs of the Lemmas. Proof of Lemma 1: We shall prove Lemma 1 indirectly. Thus we suppose that for a nonnegative bounded selfadjoint operator $T$ and an allowable function $g(\lambda)$ the strong limits

$$
\begin{gathered}
V_{A}^{+}(T)=s-\lim _{t \rightarrow \infty} e^{i t A} T e^{-i t A} P_{A}=0, \\
V_{g(A)}^{+}(T)=s-\lim _{t \rightarrow \infty} e^{i t g(A)} T e^{-i t g(A)} P_{A}=S
\end{gathered}
$$

exist with $S \neq 0$, and from these assumptions we construct a contradiction. It is obvious that $S$ is also a nonnegative bounded selfadjoint operator with $S=S P_{A}=P_{A} S \in\{g(A)\}^{\prime}$ by (2.3) to (2.5). By $S \neq 0$ it exists a $u \in P_{A} \mathscr{g}$ with $S u \neq 0$. From the definition of the allowable functions $g(\lambda)$ it follows that there is a finite interval $\Delta \subset(-\infty, \infty)$ such that $P_{A}(\Delta) u \neq 0, S P_{A}(\Delta) u \neq 0$ and $g^{\prime}(\lambda)$ is continuous, positive and of bounded variation on $\Delta$. For a nonnegative operator $S$ it follows from $S v \neq 0$ that also $(v, S v) \neq 0$. Hence we have $\left(P_{A}(\Delta) u, S P_{A}(\Delta) u\right) \neq$ 0 and then $Q S Q \neq 0$ where $Q$ is the orthogonal projection on the subspace $\mathscr{H}_{1}=\overline{\operatorname{sp}}\left\{P_{A}\left(\Delta^{\prime}\right) u, \Delta^{\prime} \subset \Delta\right\}$. It is $Q \in\{A\}^{\prime}$ and therefore $Q \in$ $\{g(A)\}^{\prime}$. By $S \in\{g(A)\}^{\prime}$ we obtain $Q S Q \in\{g(A)\}^{\prime}$. Since $g(\lambda)$ is strictly increasing on $\Delta$ it is clear that $\{Q A Q\}^{\prime}=\{Q g(A) Q\}^{\prime}$. From this identity and $Q S Q \in\{g(A)\}^{\prime}$ we finally obtain $Q S Q \in\{A\}^{\prime}$. Furthermore, we have $E(\Delta) \in\{A\}^{\prime}$, where $E(\Delta)$ is the spectral measure of $Q S Q$. We choose a $\alpha>0$ such that $E(0, \alpha)<Q$. With $R \equiv(Q-E(0, \alpha)) \in\{A\}^{\prime}$ and (5.1), (5.2) we find

$$
V_{A}^{+}(R T R)=0, V_{g(A)}^{+}(R T R)=R S R \neq 0 .
$$

$R S R$ is a nonnegative selfadjoint operator with the spectrum $\delta \in 0 \cup$ $[a, b](0<a<b<\infty)$. Now we consider continuous functions $f(\lambda)$ which are 1 on $[a, b]$ and 0 in a neighborhood of 0 .

By (2.6) and (5.3) we find

$$
V_{A}^{+}(f(R T R))=0, V_{g(A)}^{+}(f(R T R))=f(R S R)=R .
$$

From the independence of the right sides of these $f(\lambda)$ it can easily be shown that (5.4) is also true for the step-function

$$
f(\lambda)= \begin{cases}1 & \text { on }\left[a_{1}, b_{1}\right]\left(0<a_{1}<a<b<b_{1}<\infty\right) \\ 0 & \lambda \notin\left[a_{1}, b_{1}\right]\end{cases}
$$

Hence we have

$$
V_{A}^{+}(P)=0, V_{g(A)}^{+}(P)=R
$$


where $P=f(R T R)$ is an orthogonal projection with $P<R$. $\mathscr{H}_{1}$ reduces $A, P, R$ and $P, R$ are distinct from 0 only on $\mathscr{H}_{1}$. Thus it is sufficient to consider (5.5) in $\mathscr{H}_{1}$. A restricted on $\mathscr{C}_{1}$ is an operator with a simple absolutely continuous spectrum $\sigma \subset \Delta$. Then we may identify $\mathscr{H}_{1}$ with $\mathscr{L}^{2}(\sigma)$ and $a$ restricted on $\mathscr{C}_{1}$ with the multiplication operator by $\lambda$ and regard $\mathscr{H}_{1} \cong \mathscr{L}^{2}(\sigma)$ as a subspace of the large Hilbert space $\mathscr{L}^{2}(\Delta)$. In $\mathscr{L}^{2}(\Delta)$ we may identify $R$ with the multiplication operator by $\chi_{\bar{\rho}}(\lambda)$, where $\chi_{\bar{\rho}}(\lambda)$ is the characteristic function on $\bar{\rho} \subset \sigma$ with $|\bar{\rho}| \neq 0 . \quad H$ denotes the multiplication operator by $\lambda$ in $\mathscr{L}^{2}(\Delta)$. Then we obtain from (5.5)

$$
\begin{gathered}
\lim _{t \rightarrow \infty}\left\|P e^{-i H t} \psi\right\|=0 \text { for every } \psi \in \mathscr{L}^{2}(\Delta), \\
\lim _{t \rightarrow \infty}\left\|P e^{-i g(H) t} \psi\right\|=\left\|\chi_{\bar{\rho}} \psi\right\| .
\end{gathered}
$$

For the sake of simplicity, we shall assume that $\Delta=[0,2 \pi]$. We can write $g(\lambda)=\alpha \cdot \bar{g}(\lambda)+\beta$, where $\bar{g}(0)=0, \bar{g}(2 \pi)=2 \pi$, and $\alpha, \beta$ are real numbers with $\alpha>0$. Then we put

$$
\varphi_{n}(\lambda) \equiv \frac{1}{\sqrt{2 \pi}} e^{-i \lambda n}, \psi_{n}(\lambda) \equiv \frac{1}{\sqrt{2 \pi}} \sqrt{\overline{\bar{g}^{\prime}(\lambda)} e^{-i n \bar{g}(\lambda)}} .
$$

It is easy to verify that both $\varphi_{n}$ and $\psi_{n}$ form a complete orthonormal family in $\mathscr{L}^{2}(\Delta)$. Furthermore, we have $\left\|\chi_{\bar{\rho}} \psi_{n}\right\|^{2}=C>0$. With these notations we easily obtain from $(5,6),(5.7)$

$$
\begin{gathered}
\left\|P \Phi_{n}\right\|^{2}=\varepsilon_{n} \longrightarrow 0 \text { as } n \longrightarrow \infty \\
\left\|P \psi_{n}\right\|^{2}-C=\alpha_{n} \longrightarrow 0 \text { as } n \longrightarrow \infty .
\end{gathered}
$$

We set $\psi_{s}(\lambda)=\sum_{m} a_{s}^{m} \varphi_{m}(\lambda)$. Now we consider the functions $\psi_{s}(\lambda)$ for which

$$
\left\|\psi_{s}-\sum_{n=1}^{N} a_{s}^{m} \varphi_{m}\right\|^{2} \leqq \frac{C}{2}
$$

with fixed $N>0$. For the functions $\bar{\psi}_{s}(\lambda)=\left(\sqrt{\bar{g}^{1}(\lambda)}\right)^{-1} \psi_{s}(\lambda)$ by Lemma 2 we have

$$
\left\|\bar{\psi}_{\delta}-\sum_{m=\left[s q_{1}\right]-p}^{\left[s q_{2}\right]-p} \bar{a}_{s}^{m} \varphi_{m}\right\| \leqq \varepsilon
$$

where $q_{1}, q_{2}$ are positive real numbers independent of $s$, $\approx$ and $p$ is a natural number independent of $s$. It is clear that then also

$$
\left\|\psi_{s}-\sum_{m=\left[s q_{1}\right]-p^{\prime}}^{\left[s q_{2}\right]+p^{\prime}} a_{s}^{m} \varphi_{m}\right\|^{2} \leqq \frac{C}{2},
$$

with an appropriate $p^{\prime}$ independent of $s$. An elementary computation 
shows that for all $\psi_{s}$ with

$$
s \in\left(\left[\frac{1+p^{\prime}}{q_{1}}\right],\left[\frac{1+p^{\prime}}{q_{1}}\right]+1, \cdots,\left[\frac{N-p^{\prime}}{q_{2}}\right]\right)
$$

the inequality (5.10) is true.

Hence there are natural numbers $N_{1}, s_{1}$ and an $\alpha>0$ such that for every fixed $N$ with $N>N_{1}$ and $s \in\left(s_{1}, s_{1}+1, \cdots,[\alpha N]+s_{1}\right) \psi_{s}$ satisfies (5.10). Now we consider the sum

$$
S_{N}=\sum_{n=1}^{N} \sum_{s=s_{1}}^{[\alpha N]+s_{1}}\left|\left(\varphi_{n}, \psi_{s}\right)\right|^{2}
$$

and introduce the orthogonal projection $\bar{P}=1-P$. Then

$$
\begin{aligned}
& S_{N}=\sum_{n=1}^{N} \sum_{s=s_{1}}^{[\alpha N]+s_{1}}\left|\left(p \varphi_{n}, \psi_{s}\right)+\left(\varphi_{n}, \bar{p} \psi_{s}\right)\right|^{2} \\
& \leqq \sum_{n=1}^{N} \sum_{s=s_{1}}^{[\alpha N]+s_{1}}\left\{\left|\left(p \varphi_{n}, \psi_{s}\right)\right|^{2}+\left|\left(\varphi_{n}, \bar{p} \psi_{s}\right)\right|^{2}+2\left|\left(p \varphi_{n}, \psi_{s}\right)\right| \cdot\left|\left(\varphi_{n}, \bar{p} \psi_{s}\right)\right|\right\} \\
& \leqq \sum_{n=1}^{N} \sum_{s=s_{1}}^{\left[\beta \beta_{1}^{N]+s_{1}}\right.}\left\{\left|\left(p \varphi_{n}, \psi_{s}\right)\right|^{2}+\left|\left(\varphi_{n}, \bar{p} \psi_{s}\right)\right|^{2}\right\} \\
& +2 \sqrt{\left(\sum_{n=1}^{N} \sum_{s=s_{1}}^{[\alpha N]+s_{1}}\left|\left(p \varphi_{n_{1}} \psi_{s}\right)\right|^{2}\right)\left(\sum_{n=1}^{N}\left[\sum_{s=s_{1}}^{[\alpha N]+s_{1}}\left|\left(\varphi_{n_{1}} \bar{p} \psi_{s}\right)\right|^{2}\right)\right.} \\
& \leqq \sum_{n=1}^{N}\left\|P \varphi_{n}\right\|^{2}+\sum_{s=s_{1}}^{[\alpha N]+s_{1}}\left\|\bar{P} \psi_{s}\right\|^{2} \\
& +2 \sqrt{\left(\sum_{n=1}^{N}\left\|P \varphi_{n}\right\|^{2}\right)\left(\sum_{s=s_{1}}^{[\alpha N]+s_{1}}\left\|\bar{P} \psi_{s}\right\|^{2}\right)} \text {. }
\end{aligned}
$$

By (5.9) we have $\left\|\bar{P} \psi_{s}\right\|^{2}=1-C-\alpha_{s}$ and with (5.8), (5.12)

$$
\begin{aligned}
S_{N} \leqq & \sum_{n=1}^{N} \varepsilon_{n}+[\alpha N](1-C)-\sum_{s=s_{1}}^{\left[\alpha{ }^{N]+s_{1}}\right.} \alpha_{s} \\
& +2 \sqrt{\left(\sum_{n=1}^{N} \varepsilon_{n}\right)\left(1-C-\sum_{s=s_{1}}^{[\alpha N]+s_{1}} \alpha_{s}\right)} .
\end{aligned}
$$

On the other hand, by (5.10) we find

$$
S_{N} \geqq[\alpha N]\left(1-\frac{C}{2}\right) .
$$

Combining (5.13), (5.14) we obtain

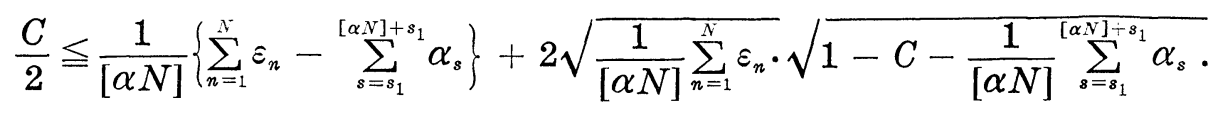

Since $\varepsilon_{n}, \alpha_{n}$ are zero sequences, also

$$
\gamma_{N}=\frac{1}{[\alpha N]} \sum_{n=1}^{N} \varepsilon_{n}, \quad \delta_{N}=\frac{1}{[\alpha N]} \sum_{s=s_{1}}^{[\alpha N]+s_{1}} \alpha_{s}
$$


are zero sequences. Hence for sufficiently large $N$ the last inequality which we have got from the assumption $S \neq 0$ is not true, which proves Lemma 1.

LEMMA 2. Let $g(\lambda)$ be a real-valued function and $g^{\prime}(\lambda)$ a continuous positive function on $J=[0,2 \pi]$. Then the functions $\bar{\psi}_{s}(\lambda) \epsilon$ $\mathcal{P}^{2}(\Delta)$ defined by

$$
\bar{\psi}_{s}(\lambda) \equiv \frac{1}{\sqrt{2 \pi}} e^{-i s g(\lambda)}=\sum_{m=-\infty}^{\infty} \bar{a}_{s}^{n} \frac{1}{\sqrt{2 \pi}} e^{-i \lambda m}
$$

possess the following properties: For every $\varepsilon$ with $0<\varepsilon<1$ and every natural nnmber $s>0$ there exist two real positive numbers $q_{1}, q_{2}$ independent of $s, \varepsilon$ and a natural number $p$ independent of $s$ such that

$$
\sum_{m=\left[s q_{1}\right]-p}^{\left[s q_{2}\right]+p}\left|\bar{a}_{s}^{m}\right|^{2} \geqq 1-\varepsilon
$$

Proof. Let $\alpha_{1}=\min _{\lambda \in \Delta} g^{\prime}(\lambda), a_{2}=\max _{\lambda \in \Delta} g^{\prime}(\lambda)$. Let $s$ be a fixed natural number. We consider integral numbers $m$ with $m>s \cdot a_{2}$ or $s \cdot a_{1}>m$. For these $m$ we have $\left|s \cdot g^{\prime}(\lambda)-m\right|>0$ and we can write

$$
\bar{a}_{s}^{m}=\frac{1}{2 \pi} \int_{0}^{2 \pi} d \lambda e^{-i s \cdot g(\lambda)} e^{i m \lambda}=\frac{1}{2 \pi} \int_{0}^{2 \pi} d \lambda \frac{1}{-i\left(s g^{\prime}(\lambda)-m\right)}\left(\frac{d}{d \lambda} e^{-i(s g(\lambda)-m \cdot \lambda)}\right)
$$

Integrating by parts and an elementary computation shows that

$$
\begin{aligned}
\left|\bar{a}_{s}^{m}\right| \leqq & \frac{1}{2 \pi} \mid\left[\frac{1}{-i\left(s g^{\prime}(\lambda)-m\right)} e^{-i(s g(\lambda)-m \lambda)}\right]_{0}^{2 \pi} \\
& +\int_{0}^{2 \pi} e^{-i(s g(\lambda)-m \lambda)} d\left(\frac{1}{i\left(g^{\prime}(\lambda) s-m\right)}\right) \mid \\
\leqq & \frac{1}{2 \pi}\left\{\frac{2}{|s \alpha-m|}+\int_{0}^{2 \pi}\left|d\left(\frac{1}{s g^{\prime}(\lambda)-m}\right)\right|\right\} \\
\leqq & \frac{1}{2 \pi}\left\{\frac{2}{|s \alpha-m|}+\frac{M \cdot s}{|s \alpha-m|^{2}}\right\} \\
= & \frac{1}{2 \pi \mid s \alpha-m}\left\{2+\frac{M}{\left|\alpha-\frac{m}{s}\right|}\right\},
\end{aligned}
$$

where $M$ is the total variation of $g^{\prime}(\lambda)$ on $\Delta$ and $\alpha=a_{1}$ if $m<s \cdot a_{1}$ or $\alpha=a_{2}$ if $m>s \cdot a_{2}$. Let $p^{\prime}$ be a positive integral number, then by (5.17) we have 


$$
\begin{aligned}
\sum_{m=\left[s 2 a_{2}\right]+p^{\prime}+1}^{\infty}\left|\bar{\alpha}_{s}^{m}\right|^{2} & \leqq \frac{1}{(2 \pi)^{2}}\left(2+\frac{M}{a_{2}}\right)^{2} \sum_{m=\left[s 2 a_{2}\right]+p^{\prime}+1}^{\infty} \frac{1}{\left|m-\left[s a_{2}\right]-1\right|^{2}} \\
& <\frac{1}{(2 \pi)^{2}}\left(2+\frac{M}{a_{2}}\right)^{2} \sum_{n=p^{\prime}}^{\infty} \frac{1}{n^{2}}
\end{aligned}
$$

and entirely analogous

$$
\begin{aligned}
\sum_{m=\left[s(1 / 2) a_{1}\right]-p^{\prime}-1}^{-\infty}\left|\bar{a}_{s}^{m}\right|^{2} & \leqq \frac{1}{(2 \pi)^{2}}\left(2+\frac{2 M}{a_{1}}\right)^{2} \sum_{m=\left[s(1 / 2) a_{1}\right]-p^{\prime}-1}^{-\infty} \frac{1}{\left|s a_{1}-m\right|^{2}} \\
& <\frac{1}{(2 \pi)^{2}}\left(2+\frac{2 M}{a_{1}}\right)^{2} \sum_{n=-p^{\prime}}^{\infty} \frac{1}{n^{2}}
\end{aligned}
$$

where $[a]$ is the smallest integer $r>a-1$.

For sufficiently large $p^{\prime}$ from (5.18), (5.19) we find

$$
\sum_{m=\left[s 2 a_{2}\right]+p^{\prime}+1}^{\infty}\left|\bar{a}_{s}^{m}\right|^{2}+\sum_{m=\left[s(1 / 2) a_{1}\right]-p^{\prime}-1}^{-\infty}\left|\bar{a}_{s}^{m}\right|^{2}<\varepsilon
$$

for all positive integral numbers $s$ and every $\varepsilon>0$. With $q_{1}=(1 / 2) a_{1}$, $q_{2}=2 \cdot a_{2}$ and by $\left|\psi_{s}\right|^{2}=\sum_{m}\left|\bar{a}_{s}^{m}\right|^{2}=1$ we finally obtain (5.16).

LEMma 3. Let $g(\lambda), \bar{\psi}_{s}(\lambda)$ be defined as in Lemma 2 and let $g^{\prime}(\lambda)$ be continuous, strictly monotone on 4 . Then the functions $\bar{\psi}_{s}(\lambda)$ possess the following properties: For every $\varepsilon$ with $0<\varepsilon<1$ there exists an $N$ such that for all integral numbers $m, s$ with $s>N$

$$
\left|\bar{a}_{s}^{m}\right|<\varepsilon \text {. }
$$

Proof. From the continuity and strict monotony of the positive function $g^{\prime}(\lambda)$ on $\Delta$ it follows that for every real number $x$ and $\varepsilon>0$ there is an interval $\Delta_{x} \leqq \Delta$ of the length $l_{x} \leqq \varepsilon \cdot \pi$ such that

$$
\alpha(\varepsilon) \equiv \min _{x \in R_{1}}\left(\min _{\lambda \in \Delta-\Delta_{x}}\left|g^{\prime}(\lambda)+x\right|\right)
$$

exists and $\alpha(\varepsilon)>0$. Hence with $x=-m / s$ we have

$$
\begin{aligned}
\left|\bar{a}_{s}^{m}\right| & =\left|\frac{1}{2 \pi} \int_{0}^{2 \pi} d \lambda e^{-i s g(\lambda)} e^{i m \lambda}\right| \\
& \leqq \frac{\varepsilon}{2}+\frac{1}{2 \pi}\left|\int_{\Delta-\Delta x} d \lambda e^{-i s(g(\lambda)-x \lambda)}\right| .
\end{aligned}
$$

The domain of integration $\left(\Delta-\Delta_{x}\right)$ consists of one or two intervals in dependence on $x$ and $\varepsilon$. Let $\Delta^{\prime}=[a, b] \leqq \Delta$ be such an interval. Then

$$
\left|\int_{a}^{b} d \lambda e^{-i s(g(\lambda)-x \lambda)}=\right| \int_{a}^{b} \frac{d \lambda}{-i s\left(g^{\prime}(\lambda)-x\right)}\left(\frac{d}{d \lambda} e^{-i s(g(\lambda)-x \lambda)}\right) \mid
$$




$$
\leqq\left|\left[\frac{1}{-i s\left(g^{\prime}(\lambda)-x\right)} e^{-i s(g(\lambda)-x \lambda)}\right]_{a}^{b}+\int_{a}^{b} e^{-i s(g(\lambda)-x \lambda)} d\left(\frac{1}{i s\left(g^{\prime}(\lambda)-x\right)}\right)\right|
$$

(5.23) $\leqq \frac{2}{s \cdot \alpha}+\int_{a}^{b}\left|d\left(\frac{1}{s\left(g^{\prime}(\lambda)-x\right.}\right)\right|$

$$
\leqq \frac{2}{s \cdot \alpha}+\frac{M}{s \cdot \alpha}
$$

where $\alpha$ is defined by (5.21) and $M=\left|g^{\prime}(b)-g^{\prime}(a)\right|$. From (5.22) and (5.23) we have

$$
\left|a_{s}^{m}\right| \leqq \frac{\varepsilon}{2}+\frac{1}{\pi s} \cdot \frac{2+M}{\alpha} .
$$

If we put $N=(2 / \varepsilon \cdot \pi) \cdot \frac{2+M}{\alpha}$, then this implies $(5.20)$.

ACKNowledgment. It is a pleasure to thank Dr. H. Baumärtel for suggesting this problem and for many stimulating discussions.

\section{REFERENCES}

1. H. Baumgärtel, $Z u$ einem Problem von M. G. Krein, Mathematische Nachrichten, 58 (1973), 279-294.

2. T. Kato, Perturbation Theory for Linear Operators, Springer-Verlag, Berlin, 1966.

3. T. Kato and S. T. Kuroda, The abstract theory of scattering, The Rocky Mountain

J. of Math., 1 (1971), 127-171.

Received August 27, 1974.

Akademie Der Wissenschaften Der DDR 



\section{PACIFIC JOURNAL OF MATHEMATICS}

\section{EDITORS}

RICHARD ARENS (Managing Editor) University of California

Los Angeles, California 90024

\section{R. A. Beaumont}

University of Washington Seattle, Washington 98105
J. DugundJI Department of Mathematics University of Southern Californıa Los Angeles, California 90007

D. Gilbarg aNd J. Milgram Stanford University Stanford, California 94305

\section{ASSOCIATE EDITORS}

E. F. BeCKENBACH

B. H. NeUmanN

F. Wolf

K. YoSHIDA

\section{SUPPORTING INSTITUTIONS}

UNIVERSITY OF BRITISH COLUMBIA CALIFORNIA INSTITUTE OF TECHNOLOGY UNIVERSITY OF CALIFORNIA MONTANA STATE UNIVERSITY UNIVERSITY OF NEVADA NEW MEXICO STATE UNIVERSITY OREGON STATE UNIVERSITY UNIVERSITY OF OREGON OSAKA UNIVERSITY
UNIVERSITY OF SOUTHERN CALIFORNIA STANFORD UNIVERSITY UNIVERSITY OF TOKYO UNIVERSITY OF UTAH WASHINGTON STATE UNIVERSITY UNIVERSITY OF WASHINGTON AMERICAN MATHEMATICAL SOCIETY NAVAL WEAPONS CENTER 


\section{Pacific Journal of Mathematics \\ Vol. 59, No. 1 \\ May, 1975}

Shashi Prabha Arya and M. K. Singal, More sum theorems for topological

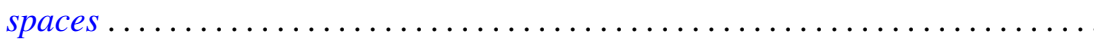

Goro Azumaya, F. Mbuntum and Kalathoor Varadarajan, On M-projective and

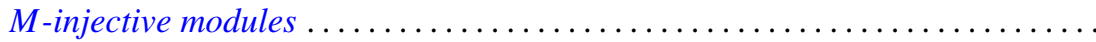

Kong Ming Chong, Spectral inequalities involving the infima and suprema of

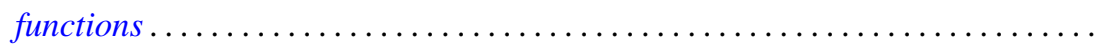

Alan Hetherington Durfee, The characteristic polynomial of the monodromy .......

Emilio Gagliardo and Clifford Alfons Kottman, Fixed points for orientation

preserving homeomorphisms of the plane which interchange two points ......

Raymond F. Gittings, Finite-to-one open maps of generalized metric spaces .......

Andrew M. W. Glass, W. Charles (Wilbur) Holland Jr. and Stephen H. McCleary,

$a^{*}$-closures of completely distributive lattice-ordered groups .............

Matthew Gould, Endomorphism and automorphism structure of direct squares of

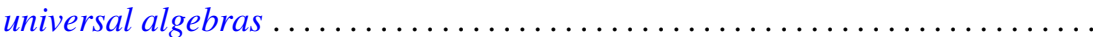

R. E. Harrell and Les Andrew Karlovitz, On tree structures in Banach spaces .....

Julien O. Hennefeld, Finding a maximal subalgebra on which the two Arens

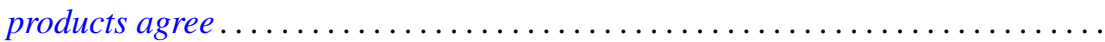

William Francis Keigher, Adjunctions and comonads in differential algebra .......

Robert Bernard Kelman, A Dirichlet-Jordan theorem for dual trigonometric

series

Allan Morton Krall, Stieltjes differential-boundary operators. III. Multivalued operators-linear relations...

Hui-Hsiung Kuo, On Gross differentiation on Banach spaces .

Tom Louton, A theorem on simultaneous observability ...

Kenneth Mandelberg, Amitsur cohomology for certain extensions of rings of algebraic integers.

Coy Lewis May, Automorphisms of compact Klein surfaces with boundary . . .

Peter A. McCoy, Generalized axisymmetric elliptic functions .

211

Muril Lynn Robertson, Concerning Siu's method for solving $y^{\prime}(t)=F(t$, $y(g(t)))$. .

Richard Lewis Roth, On restricting irreducible characters to normal

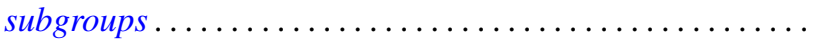

Albert Oscar Shar, $P$-primary decomposition of maps into an $H$-space .

Kenneth Barry Stolarsky, The sum of the distances to certain pointsets on the unit circle.

Bert Alan Taylor, Components of zero sets of analytic functions in $C^{2}$ in the unit ball or polydisc

Michel Valadier, Convex integrands on Souslin locally convex spaces ...

Januario Varela, Fields of automorphisms and derivations of $C$

Arnold Lewis Villone, A class of symmetric differential operators with deficiency

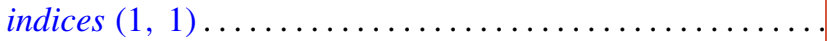

